\title{
INVESTIGACIONES
}

\section{La organización retórica del resumen o abstract del artículo de investigación en educación*}

\author{
The rhetorical organization of research article abstracts in education
}

A organização retórica de resumo de artigo de investigação em educação

\author{
Angie Quintanilla Espinoza
}

\begin{abstract}
Becaria CONICYT. Programa de Doctorado en Lingüística, Universidad de Concepción. Facultad de Ciencias de la Educación. Universidad San Sebastián. Concepción, Chile. Telf.: (41)2487201. Correo electrónico: angie.quintanilla@uss.cl
\end{abstract}

\section{RESUMEN}

El estudio de la organización-estructura del género discursivo abstract es esencial para la adecuada alfabetización académica y la consecuente divulgación de conocimiento académico-científico. Este estudio describe la organización retórica de abstracts de artículos de investigación en el área de educación. Para ello se analiza la estructura genérica de 50 textos pertenecientes a la revista Estudios Pedagógicos de la Universidad Austral de Chile, a partir de un enfoque descendente (movidas retóricas de Swales y Feak, 2009). Los resultados muestran dos patrones básicos en el 46\% de los textos que conforman el corpus (M2, M3, M4, (M5)), mientras que el resto no sigue un patrón prefijado consistente. Esto sugiere que es necesario fortalecer las competencias discursivas al interior de las comunidades académico-científicas. retóricas.

Palabras clave: abstract de artículo de investigación, análisis de género, alfabetización académica, movidas

\section{ABSTRACT}

The study of organization-structure of abstracts as an academic genre is essential for proper academic literacy and the consequent dissemination of scientific knowledge. This study describes the rhetorical organization of abstracts of research articles in the area of education. In order to do this, the generic structure of 50 texts from Estudios Pedagógicos magazine from Universidad Austral de Chile, is analyzed using a top-down approach (Rhetorical Moves of Swales and Feak, 2009). The results show two basic patterns in $46 \%$ of the texts that make up the corpus (M2, M3, M4, (M5), while the rest do not follow a consistent preset pattern. This suggests the need to strengthen the discourse skills within the academic and scientific communities.

Key words: research article abstract, genre analysis, academic literacy, rhetorical moves. 


\section{INTRODUCCIÓN}

Es innegable el rol que cumple el abstract de artículos de investigación (AI) en el mundo científico como un medio de difusión de la creación de nuevo conocimiento. Por otro lado, la mayoría de las personas ya sean expertos o principiantes harán un primer contacto con un AI a partir de la lectura de su abstract, y decidirán (cuando el tema sea de su interés) si continuar o no con la lectura del artículo dependiendo de la claridad con la cual el autor exponga su estudio en este primer párrafo.

Es en este contexto que estudiar la forma en la cual se configura el discurso académico permite conocer las convenciones que se han establecido en sus distintos géneros discursivos. De acuerdo a Sánchez (2012) el desconocimiento de estas convenciones y sus sub-tipologías textuales es una de las problemáticas generales que se dan en la escritura académica. Esto, de acuerdo al autor, se evidencia en una estructura poco ajustada a la establecida en dicho género, entre estas una inadecuada organización-estructura del resumen. Esta problemática, denominada alfabetización académica y/o lingüística superior, no es tratada en profundidad en los procesos de formación universitaria. En general, los investigadores-autores desarrollan esta alfabetización a partir de su propia experiencia en la disciplina y en el ámbito profesional.

Si se pretende desarrollar una alfabetización académica superior es necesario conocer la estructura de las convenciones del género académico, y en lo que concierne a esta investigación, la organización retórica del abstract o resumen en el área de educación.

Atendiendo a estos requerimientos, el estudio que se presenta a continuación tiene como objetivo describir la estructura retórica de los abstract de AI en el área de la educación a través del modelo de movidas retóricas de Swales y Feak (2009).

A través de este estudio se espera también dar respuesta a las siguientes preguntas de investigación: ¿Cómo se configura la organización retórica del abstract en artículos de investigación en educación? ¿Qué elementos léxicos y combinaciones tiempo-aspecto gramatical ocurren en el abstract en artículos de investigación en educación?

El presente artículo está organizado en las siguientes secciones: En la sección 1 se presenta el marco teórico: el abstract o resumen y el análisis de movidas retóricas. En la sección 2 se describe el corpus y la metodología de la investigación. En la sección 3 se presentan los resultados del estudio. Finalmente, en la sección 4 se presentan las conclusiones y discusión final.

\section{MARCO TEÓRICO}

\subsection{ABSTRACT O RESUMEN}

En esta investigación se ha fijado como objeto de estudio el género abstract en artículos de investigación (AI) en el área de educación. Este subtipo de texto tiene como función sintetizar las ideas centrales de un artículo y proveer a los lectores de un primer contacto con el AI que está siendo presentado.

Una de las normas de publicación más ampliamente utilizada en investigación, APA (American Psychological Association), en su publicación (2010: 26) señala que "A wellprepared abstract can be the most important single paragraph in an article". La importancia 
de este "párrafo" radica en que a partir de su lectura, el lector decide si leer o no el artículo.

El manual de publicación APA (2010) establece que un abstract de un estudio empírico debe poseer 5 componentes:

1. Problema de investigación.

2. Participantes.

3. Características esenciales de la metodología de estudio.

4. Resultados.

5. Conclusiones e implicaciones o aplicaciones.

Desde esta misma perspectiva, los autores Paltridge y Starfield (2007) señalan que, en general, el objetivo del abstract es proveer un resumen de un estudio dando respuesta a las siguientes interrogantes:

- ¿Cuál fue el propósito general del estudio?

- ¿Cuál fue el objetivo específico del estudio?

- ¿Por qué se realizó el estudio?

- ¿Cómo se llevó a cabo el estudio?

- ¿Qué reveló el estudio?

Y que por esta razón, la estructura usual de un abstract en AI sería la siguiente:

- Introducción del tema.

- Objetivos o propósito de la investigación.

- Método de investigación.

- Resultado final.

- Conclusiones principales.

Los planteamientos hasta aquí presentados no pretenden ejemplificar cuál es la estructura ideal de un abstract en AI. Sin embargo muchos autores (Hyland, 2000; Santos, 1996; Swales y Feak, 2009; Weissberg y Buker, 1990) plantean que este género se caracteriza por una estructura de cinco movidas retóricas básicas, las que poseen una relación directa con los componentes que deben estar presentes en un abstract de AI como los señalados por Paltridge y Starfield (2007): introducción, objetivo de la investigación, método utilizado, resultados y conclusiones.

\subsection{EL ANÁLISIS DE MOVIDAS RETÓRICAS EN EL ABSTRACT DE AI}

El enfoque de movidas retóricas y pasos, propuesto por Swales $(1990,2004)$ ha tenido gran relevancia en el estudio de la organización retórica de textos académicos. El propósito inicial de este autor fue analizar y describir la estructura de las introducciones presentes en artículos científicos para de este modo establecer regularidades (tendencias) e identificar ejemplares de un género.

Una movida o "move" puede ser definida de acuerdo a Swales (2004: 228) como "a discoursal and rhetorical unit that performs a coherent communicative function in a written or spoken discourse". Cada una de estas movidas posee un propósito comunicativo espe- 
cífico, el cual se asocia a una unidad discursiva que contribuye al propósito general del género (Swales, 1990).

A partir de la propuesta de Swales se han desarrollado numerosas investigaciones en el ámbito del análisis de géneros académicos, tanto en sus propósitos comunicativos como en las estrategias retóricas utilizadas (Biber, Connor y Upton, 2007; Hyland, 2000; Ibañez, 2010; Parodi, 2008; Samraj, 2002, 2005; Santos, 1996; Weissberg y Buker, 1990).

Con respecto a la estructura del abstract del AI, las investigaciones realizadas han dado como resultado el siguiente patrón de cuatro movidas retóricas: introducción, metodología, resultados y discusión (Bhatia, 1993; Swales, 1981, 1990). Otros autores ya mencionados (Hyland, 2000; Santos, 1996; Swales y Feak, 2009; Weissberg y Buker, 1990) proponen una movida adicional en la cual el autor sitúa la investigación desde un marco referencial teórico o presenta el contexto y la motivación del estudio. A partir de esto, se han desarrollado algunos de los modelos para la clasificación de las movidas retoricas en abstracts de AI que se presentan a continuación.

\section{Modelo de Weissberg y Buker (1990)}

Este modelo (Tabla 1) subdivide la introducción en dos movidas retóricas: la primera, que introduce antecedentes del estudio y la segunda que presenta el propósito principal y el alcance de la investigación.

Tabla 1. Clasificación de movidas retóricas (Weissberg y Buker, 1990)

\begin{tabular}{l} 
ORDEN DE LOS ELEMENTOS TÍPICOS INCLUIDOS EN UN ABSTRACT \\
\hline$B=$ algunos antecedentes \\
$P=$ la actividad principal (o propósito) del estudio y su alcance \\
$M=$ información acerca de la metodología utilizada en el estudio \\
$R=$ los resultados más importantes del estudio \\
$C=$ una declaración de conclusión o recomendación
\end{tabular}

\section{Modelo de Hyland (2000)}

En este modelo, el autor postula que en la introducción el autor contextualiza su investigación y explica las razones que motivan su trabajo. Esto correspondería a la movida B (background) de la estructura de Weissberg y Buker (1990). Tanto la introducción como los antecedentes tienen como propósito situar la investigación del autor (Piqué-Noguera, 2012). 
Tabla 2. Clasificación de movidas retóricas (Hyland, 2000)

\begin{tabular}{ll}
\hline \multicolumn{1}{c}{ MOVIDA } & \multicolumn{1}{c}{ FUNCIÓN } \\
\hline Introducción & Establece el contexto del artículo y motiva la investigación o discusión. \\
Propósito & Indica propósito, tesis o hipótesis, delimita la intención detrás del artículo. \\
Método & Provee información del diseño, procedimientos, suposiciones, enfoque, datos, etc. \\
Producto & Expone los principales hallazgos o resultados, el argumento, o lo que se logró. \\
Conclusiones & $\begin{array}{l}\text { Interpreta o extiende los resultados más allá del alcance del artículo, saca conclu- } \\
\text { siones, señala aplicaciones o implicaciones mayores. }\end{array}$ \\
\hline
\end{tabular}

\section{Modelo de Swales y Feak (2009)}

Este modelo es bastante similar al propuesto por Hyland (2000) e incorpora diferentes terminologías en sus etiquetas como una forma de agrupar algunos de los modelos que se han propuesto. Además, incorpora algunas de las interrogantes que deben ser respondidas en cada una de las movidas.

Tabla 3. Clasificación de movidas retóricas (Swales y Feak, 2009)

\begin{tabular}{lll}
\hline MOVIDAS & \multicolumn{1}{c}{ ETIQUETAS TÍPICAS } & \multicolumn{1}{c}{ PREGUNTAS IMPLICADAS } \\
\hline Movida 1 & antecedentes/ introducción/ situación & ¿Qué sabemos acerca del tema? \\
Movida 2 & presentar investigación/ propósito & ¿De qué se trata el estudio? \\
Movida 3 & métodos/ materiales/ sujetos/ procedimientos & ¿Cómo fue hecho? \\
Movida 4 & resultados/ hallazgos & ¿Qué fue descubierto? \\
Movida 5 & discusión/ conclusión/ implicación/ aplicaciones & ¿Qué significan los hallazgos? \\
\hline
\end{tabular}

Los tres modelos presentados resumen de alguna manera la investigación que se ha realizado con respecto a la organización retórica de los abstracts en AI a partir del enfoque de las movidas retóricas desarrollado por Swales (1990).

\section{DESCRIPCIÓN DEL CORPUS Y METODOLOGÍA}

El corpus de esta investigación está compuesto por 50 abstracts de la Revista Estudios Pedagógicos de la Facultad de Filosofía y Humanidades de la Universidad Austral de Chile, correspondientes a los tres últimos números, publicados en el año 2013. Esta revista fue seleccionada por ser en este momento la única en la temática (en Chile) con indexación Scielo y SCOPUS, además de poseer un SJR (Scientific Journal Rankings) de 0.22. 
En el presente estudio se utiliza el modelo de Swales y Feak (2009) para analizar y clasificar las movidas retóricas presentes en los abstracts del AI en educación. Este análisis se realiza en base a la función o contenido del texto utilizando un enfoque descendente o top-down.

Una vez que las movidas fueron identificadas se procedió a realizar cálculos simples de frecuencia con la finalidad de conocer cuáles son las movidas que se utilizan con mayor frecuencia. Luego se estableció el orden de ocurrencia de dichas movidas en cada uno de los textos que formaban parte del corpus con el propósito de caracterizar la organización retórica del abstract de AI en educación. Finalmente, se agrupan las movidas de acuerdo a su tipo y se buscan patrones lingüísticos comunes que describan dichas movidas retóricas.

\section{RESULTADOS}

\subsection{DESCRIPCIÓN CUANTITATIVA}

A continuación se desarrolla la descripción cuantitativa de los resultados del presente estudio.

\subsubsection{Análisis de datos básicos}

La Tabla 4 presenta estadísticas básicas del corpus utilizado en esta investigación, las cuales se han obtenido a través de los programas TextStat (Simple Text Analysis Tool) y scp 4.09 (Simple Concordance Program).

Tabla 4. Estadísticas básicas del corpus

\begin{tabular}{lr}
\hline $\mathrm{N}^{\circ}$ de párrafos & 50 \\
\hline $\mathrm{N}^{\circ}$ de palabras & 6646 \\
$\mathrm{~N}^{\circ}$ de oraciones & 222 \\
$\mathrm{~N}^{\circ}$ de palabras por oración & 29.23 \\
$\mathrm{~N}^{\circ}$ de palabras por párrafo & 132.92 \\
Types/tokens & 0.24164735 \\
Types/sqrt(tokens) & 20.69297297 \\
Yule's k & 172.78037637 \\
Índice de legibilidad (Flesch) & -31.42 \\
\hline
\end{tabular}

Esta información de tipo cuantitativa muestra la densidad léxica de los abstracts utilizados, especialmente type-token y types/sqrt(tokens), estas dos medidas permiten determinar la diversidad del vocabulario utilizado en el corpus. 
La longitud de las oraciones (29.23 palabras por oración) también es una medida de la claridad y comprensibilidad del texto. De acuerdo a MacDonald (1990) el promedio de palabras por oración en escritura académica es de 23.26. También se presenta el índice de legibilidad el cual muestra la facilidad de comprensión de un texto, en este caso corresponde a -31.42, lo cual indica que este texto es de difícil comprensión y correspondería a un nivel adecuado para académicos y conocedores de la disciplina.

Otro aspecto a considerar es el número de palabras por abstract (132.92). En general, los artículos comprenden entre 100 y 250 de acuerdo a las normas internacionales (Kaplan et al., 1994). En el caso de la revista estudiada, la indicación a los autores señala que la extensión del abstract no debe exceder las 150 palabras.

\subsubsection{Frecuencia de movidas}

El proceso de cuantificación de las movidas retóricas evidenció que de las cinco movidas presentes en el modelo de Swales y Feak (2009) solo tres se presentan con mayor frecuencia. Como se aprecia en el Gráfico 1, se destaca M2 (propósito) con un 90\% de ocurrencia, le sigue M4 (resultados) 80\% y M3 (método) con un 72\%.

Las movidas con menor ocurrencia son M5 (conclusión) y M1 (introducción) con un $42 \%$ y $34 \%$ respectivamente.

Gráfico 1. Ocurrencia promedio de las movidas

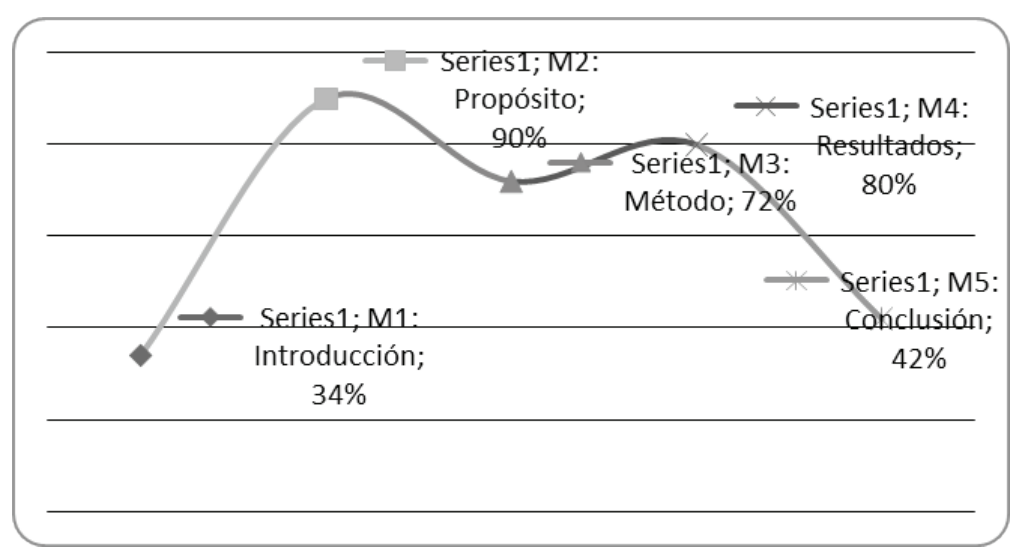

\subsubsection{Estructura (orden) de las movidas}

$\mathrm{Al}$ analizar el orden en el cual se presentaban las movidas retóricas en cada uno de los abstracts estudiados (Anexo 1), la estructura ideal presente en los modelos de Weissberg y Buker (1990), Hyland (2000) y Swales y Feak (2009) solo ocurre en uno de los textos (2\%). Sin embargo, se presenta la estructura M2, M3, M4 en un 20\% de estos y una variación de esta estructura (M2, M4, M3 y M2, M4, M3) en un 4\% del corpus. Finalmente, se observa la estructura M2, M3, M4, M5 en un 22\% de los abstracts analizados (Tabla 5). 
Tabla 5. Estructura de las movidas

\begin{tabular}{lcc}
\hline \multicolumn{1}{c}{ Estructura } & $\mathrm{N}^{\circ}$ de ocurrencias & Porcentaje \\
\hline M1,M2,M3,M4,M5 & 1 & $2 \%$ \\
M2,M3,M4 & 10 & $20 \%$ \\
M2, M4, M3 & 1 & $2 \%$ \\
M3, M2, M4 & 1 & $2 \%$ \\
M2,M3,M4,M5 & 11 & $22 \%$ \\
\hline
\end{tabular}

\subsection{DESCRIPCIÓN CUALITATIVA}

A continuación se desarrolla la descripción cualitativa de los resultados del presente estudio a partir de la descripción de las movidas retóricas.

\section{Movida 1. Introducción}

Esta movida sitúa la investigación a partir de la contextualización y la presentación de antecedentes o investigaciones previas en el área de estudio. Esto generalmente se observa a partir del establecimiento de una problemática o de un vacío en las investigaciones previas realizadas en torno a la temática central presentada en el abstract. En el corpus esto se observa de la siguiente manera:

(1) La lectura eficaz se ha constituido en uno de los desafíos fundamentales del sistema educacional chileno, luego que diversas mediciones internacionales mostraran escasos logros en la competencia lectora de niños y jóvenes.

(2) Uno de los aspectos claves de la formación inicial de profesores es la participación de los estudiantes en experiencias pedagógicas en contextos reales. Estas experiencias suelen ser limitadas y parcialmente mediadas por los profesores de los programas de formación.

(3) Los programas de formación docente continua tradicionales, generalmente prescriptivos y descontextualizados del aula, han tenido bajo impacto en los procesos de transformación de las prácticas docentes.

(4) Durante los últimos años, en Chile se está produciendo un fenómeno de inmigración proveniente de otros países que está conformando un nuevo contexto social multicultural. Por supuesto, las aulas reflejan esta pluralidad de culturas, a las que debe dar respuesta, principalmente, a través de la formación del profesorado.

De los 50 abstracts analizados, solo 17 (34\%) utilizan esta movida retórica. Por lo cual se considera que M1 es más bien opcional en el corpus.

Con respecto a los tiempos verbales utilizados en esta movida retórica se observa con mayor frecuencia el presente simple, el presente perfecto y la voz pasiva. 


\section{Movida 2. Propósito}

Esta movida tiene como objetivo presentar el propósito de la investigación que se ha llevado a cabo. En el corpus esto se ejemplifica como sigue:

(1) Este artículo describe una investigación sobre las concepciones docentes acerca del uso de las Tecnologías de la Información y la Comunicación (TIC) en educación secundaria y los cambios que se generan en dichas concepciones cuando los profesores son expuestos a experiencias de interacción con herramientas Web 2.0.

(2) Este trabajo explora la conceptualización que hacen los profesores universitarios sobre sus estudiantes ...

(3) El objetivo de este artículo es describir la forma en que los estudiantes de la Facultad de Ciencias Sociales de la Universidad de Chile conceptualizan a un "buen docente".

En esta segunda movida se observa el establecimiento del propósito de la investigación a partir de construcciones similares las cuales incluyen: el objetivo/propósito de este artículo/ investigación, o bien una construcción del tipo el artículo describe/presenta. La Tabla 6 ilustra la formula y/o patrón predominante en esta movida.

Tabla 6. Patrón predominante empleado en la movida Propósito

\begin{tabular}{|c|c|c|c|}
\hline $\begin{array}{l}\text { Expresión } \\
\text { Deíctica }\end{array}$ & $\begin{array}{l}\text { Sustantivo } \\
\text { de intención }\end{array}$ & $\begin{array}{l}\text { Tipo de investigación } \\
\text { o género }\end{array}$ & $\begin{array}{l}\text { Verbos } \\
\text { introductorios }\end{array}$ \\
\hline $\begin{array}{l}\text { este (22) } \\
\text { el (18) } \\
\text { esta (5) }\end{array}$ & $\begin{array}{l}\text { objetivo (5) } \\
\text { propósito (4) }\end{array}$ & $\begin{array}{l}\text { artículo (19) } \\
\text { estudio (9) } \\
\text { trabajo (8) } \\
\text { investigación (4) } \\
\text { informe (1) } \\
\text { reporte (1) } \\
\text { síntesis (1) } \\
\text { proyecto (1) } \\
\text { experiencia (1) }\end{array}$ & $\begin{array}{l}\text { presenta (7) } \\
\text { describe (6) } \\
\text { es (5) } \\
\text { fue (5) } \\
\text { muestra (3) } \\
\text { busca (2) } \\
\text { estudia (2) } \\
\text { realiza (1) } \\
\text { discute (1) } \\
\text { caracteriza (1) } \\
\text { pretende (1) } \\
\text { aborda (1) } \\
\text { analiza (1) } \\
\text { recoge (1) } \\
\text { se analiza (1) } \\
\text { consiste en (1) } \\
\text { da cuenta de (1) } \\
\text { desarrolla (1) } \\
\text { pretendió (1) } \\
\text { se presentan (1) }\end{array}$ \\
\hline
\end{tabular}


La expresión deíctica predominante es "este". En relación con el tipo de investigación, los tres géneros más utilizados son "artículo", "estudio" y "trabajo". Los verbos introductorios que predominan son "presenta", "describe", "es" (presente simple) y "fue" (pasado simple). El tiempo verbal que prevalece es el presente simple, sin embargo, se observa el uso del pasado simple y de construcciones en voz pasiva.

\section{Movida 3. Método}

En esta movida el autor presenta la forma en que se desarrolla la investigación a partir de la descripción de los procedimientos llevados a cabo, los materiales utilizados, los sujetos involucrados en la investigación, etc. En el corpus esto se observa de la siguiente manera:

(1) Se utilizó entrevistas en profundidad y relatos escritos. La información producida, una vez transcrita, se estudió desde el análisis de contenido apoyado en el programa computacional Atlas ti 5.0 .

(2) Se trabajó con un diseño de tipo experimental.

(3) Los datos fueron recolectados en base a un instrumento de 18 preguntas...

(4) La metodología utilizada ha sido el análisis lexicográfico y de contenido de, por un lado, el principal decreto que regula la enseñanza secundaria obligatoria en España...

A pesar de que esta es una de las tres movidas con mayor ocurrencia en el abstract de AI en educación, no se observa un patrón en la forma en que se presenta esta movida, ejemplos 1, 2, 3 y 4 . Es posible que la variedad de formas lingüísticas en las que se observa se deba a la naturaleza diversa de los elementos que pueden constituir la M3-Método. A pesar de esto, es posible determinar que para reportar la metodología empleada se utiliza el pasado simple y la voz pasiva. Esto se debe a que el propósito de esta movida es explicar la metodología previamente utilizada.

\section{Movida 4. Resultados}

En esta movida se presentan los principales resultados y/o hallazgos obtenidos a partir de la investigación.

(1) Los principales resultados demuestran que si bien hay una gran diversidad en el tipo de formación recibida y en la comprensión de las ideas que tienen los docentes sobre qué es formar ciudadanos en la escuela, esto no ocurre del mismo modo en sus clases, donde las estrategias son mucho más homogéneas.

(2) Los resultados indicaron que las actitudes muestran una tendencia favorable, excepto para mantener una relación permanente o íntima.

(3) Los resultados fundamentales se orientan hacia procesos de acción y despliegue corpóreo fundamentalmente de dependencia y heteronomía, en las sesiones formales de clases, a diferencia de lo que ocurre en espacios de recreo y de libre acción.

En este caso es posible observar similitudes en la forma como se presenta la construcción de la movida por ejemplo: los resultados muestran/ indican, los resultados se orientan hacia, entre otros. La Tabla 7 ilustra la formula y/o patrón predominante en esta movida. 
Tabla 7. Patrón predominante empleado en la movida Resultados

\begin{tabular}{ll}
\hline Sustantivo de apertura & Verbos introductorios \\
\hline resultados (30) & muestran (14) \\
análisis (5) & indican (4) \\
estudio (1) & mostraron (2) \\
ejercicio (1) & permiten (2) \\
hallazgos (1) & mostró (1) \\
& evidencian (1) \\
& indica (1) \\
& permitieron (1) \\
& muestra (1) \\
& reveló (1) \\
& definen (1) \\
& evidenciaron (1) \\
& señalan (1) \\
& demuestran (1) \\
& indicaron (1) \\
& se orientan hacia (1) \\
& se interpretan (1) \\
& evidencia (1) \\
& conduce a (1) \\
\hline
\end{tabular}

Además del patrón que se presenta en la tabla 7, se observan las siguientes construcciones pasivas "se encontró" y "se evidencia", ejemplos 4 y 5 :

(4) En algunas de las formas de aproximarse a la actividad docente se encontró que están influyendo factores personales como la edad, género y tipo de universidad de egreso.

(5) Se evidencia la inexistencia de diferencias estadísticamente significativas en la percepción del liderazgo del docente de lenguaje

\section{Movida 5. Conclusión}

En esta última movida se discuten los resultados y su implicancia en el área de estudio, así como también las posibles aplicaciones y/o el alcance de los hallazgos. En el corpus esto se observa a través de las siguientes construcciones:

(6) Tal situación permite interpretar que la escuela es fundamentalmente, en los casos estudiados, un lugar de relaciones de resistencia y domesticación, más que de autonomía y construcción de las propias acciones.

(7) Se concluye que la emoción entendida como la aceptación del otro como legítimo otro en la convivencia, ha sido la piedra angular del proceso de autoevaluación vivenciado, pues ha inducido a la implicación activa, responsable y ética en dicho proceso. 
(8) Se concluye que entre los estudiantes de la muestra existen escasos rasgos de los llamados nuevos aprendices y aparece una brecha perceptual importante entre distintos contextos, donde el aprendizaje se conceptualiza desde una mirada escolarizada.

Esta movida es una de las que se presenta con menor frecuencia en el corpus (21 ocurrencias en 50 textos), sin embargo cuando aparece lo hace la mayor cantidad de las veces a partir de la construcción prototípica se concluye que, a pesar de que se pueden encontrar otra construcciones tales como es posible concluir o permite interpretar, entre otras. La Tabla 8 ilustra la fórmula y/o patrón predominante en esta movida.

Tabla 8. Patrón predominante empleado en la movida Conclusión

\begin{tabular}{lll}
\hline Marcadores de cierre & Sustantivo de apertura & Verbos introductorios \\
\hline en conclusión (2) & análisis (2) & permite (4) \\
finalmente (3) & implicancias (2) & se establece (2) \\
validación empírica (1) & conduce a (1) \\
implicaciones (2) & se encontraron (1) \\
trabajo (1) & se consideran (1) \\
& se exhiben (1) \\
& se concluye que (3) \\
& se construye (1) \\
& se pudo concluir (1) \\
& se reportan (1) \\
& se discute (2) \\
& se constató (1) \\
& se concluyen (1) \\
& se discuten (1) \\
\hline
\end{tabular}

\section{CONCLUSIÓNES Y DISCUSIÓN}

El objetivo de este estudio fue analizar la estructura retórica de los abstract de AI en el área de la educación a través del modelo de movidas retóricas de Swales y Feak (2009). Esto a partir del análisis de la ocurrencia de movidas y de la ocurrencia de estructuras (orden) de las mismas. Además de describir de forma cualitativa, a partir de ejemplos, el tipo de movidas encontradas en el corpus.

A partir de lo reducido del corpus utilizado en esta investigación no es posible hacer generalizaciones, pero sí es posible tener un primer acercamiento al género abstract de AI en educación en Chile.

En general, este estudio presentó la estructura retórica del abstract a partir de la ocurrencia de las movidas estudiadas, dando como resultado que las movidas que se encuentran más representadas en el corpus son M2, M4 y M3, propósito, resultados y métodos respectivamente. Mientras que las movidas M5 y M1 fueron las que presentan 
menos ocurrencia. Estos resultados concuerdan con los obtenidos por otros autores, en investigaciones recientes, en disciplinas como el comercio (Piqué-Noguera, 2012), la lingüística y la lingüística aplicada (Suntara y Usaha, 2013), y en diferencias culturales en la organización retórica de abstracts (Kafes, 2012).

Además de estudiar la recurrencias de las movidas, uno de los objetivos de este estudio fue caracterizar la estructura de dichas movidas retóricas en el abstract de AI. Los resultados obtenidos muestran dos estructuras básicas M2, M3, M4 (20\%) y M2, M3, M4, M5 (22\%), ambas estructuras tendrían una relación directa con las movidas que ocurren con mayor recurrencia $90 \%, 72 \%$ y $80 \%$, respectivamente. Sin embargo, se hace necesario en esta discusión considerar que existe un 4\% que no sigue el orden M2, M3, M4, sino que se utiliza una estructura M3, M2, M4 y M2, M4, M3. A pesar de que uno de los propósitos era establecer el orden de las movidas retóricas, no deja de ser interesante el hecho de que a pesar de que el orden no es el mismo en un par de casos, el abstract mantendría una estructura similar desde el punto de vista de los componentes incorporados, y por consiguiente el mensaje entregado por el autor (propósito, método y resultados).

La investigación en esta área, como lo muestra la literatura revisada en el marco teórico, presenta estas cinco movidas como la construcción de un abstract "ideal". Sin embargo, cabe considerar que al incluir el propósito de la investigación, la metodología utilizada y los resultados obtenidos se logra dar una idea clara de la investigación que se ha llevado a cabo.

Cabe señalar que solo un $46 \%$ de los abstracts analizados presentan las movidas M2, M3, M4, (M5) y que el resto no seguiría una tendencia tan clara. Esto podría deberse a que en esta investigación se utilizó un enfoque descendente y un modelo estándar para el abstract de AI.

La American Psychological Association (APA) en su publicación 2010 hace diferencia entre tipos de abstract dependiendo de la naturaleza de la investigación, como por ejemplo: reporte de estudio empírico, revisión de literatura o meta-análisis, artículo orientado a la teoría, artículo metodológico y de estudio de caso. Desde esta perspectiva, aparece como una temática digna de estudio la configuración retórica del abstract académico dependiendo del tipo de investigación que se busca presentar.

Finalmente, el análisis cualitativo de las movidas permite identificar similitudes en la forma en la cual las movidas se estructuran a nivel morfosintáctico a partir de la individualización de los sustantivos y verbos introductorios utilizados en cada una de ellas. Se observa que es posible identificar términos y estructuras que se repiten en cada una de las movidas. No obstante, en el caso de la M3 Método se identifica una mayor variedad léxica y estructural, esto podría deberse a la variedad de elementos que pueden constituir dicha movida, por ejemplo: metodología, muestra, tipo de análisis, materiales, procedimientos, entre otros.

Dada la importancia del género abstract en la difusión del conocimiento científico y la relevancia que este tiene al ser la carta de presentación de un AI, tanto en revistas especializadas como en presentaciones en congresos, conocer su estructura y enseñar a los nuevos investigadores cómo se configura este género puede tener un alto impacto en su formación profesional y académica.

Los hallazgos de este estudio exploratorio descriptivo tienen implicaciones pedagógicas que pueden verse reflejadas en el desarrollo de nuevo material para la enseñanza de la escritura académica que pueda fortalecer las competencias discursivas al interior de las comunidades académico científicas. 


\section{REFERENCIAS BIBLIOGRÁFICAS}

American Psychological Association. (2010). Manual de publicaciones de la American Psychological Association (3a ed.). México: Manual Moderno.

Bhatia, V. (1993). Analyzing Genre: Language Use in Professional Settings. London: Longman.

Biber, D., Connor, U., \& Upton, T. (2007). Discourse on the move. Using corpus analysis to describe discourse structure. Amsterdam: Benjamins.

Hyland, K. (2000). Disciplinary discourses: Social interactions in academic writing. London: Pearson.

Ibáñez, R. (2010). El Texto Disciplinar en la transmisión del conocimiento especializado. Estudios filológicos, 46, 59-80.

Kafes, H. (2012). Cultural traces on the rhetorical organization of research article abstracts. International Journal on New Trends in Education and Their Implications, 3, 207-220.

Kaplan, R.B., Cantor, S., Hagstrom, C., Kamhsi-Stein, L.D., Shiotani, Y., \& Zimmerman, C.B. (1994). On abstract writing. Text, 14(3), 401-426.

MacDonald, S.P. (1990). The literary argument and its discursive conventions. In W. Nash (Ed.), The Writing Scholar. Studies in academic discourse (pp. 31-62). Newbury Park: Sage Publications.

Paltridge, B., \& Starfield, S. (2007). Thesis and Dissertation Writing in a Second Language: A Handbook for Supervisors. London: Routledge Falmer imprint of Taylor \& Francis.

Parodi, G. (2008). La organización retórica de género Manual: ¿Una "colonia encadenada”? En G. Parodi (Ed.), Géneros Académicos y Géneros Profesionales: Accesos Discusivos para Saber y Hacer (pp. 169-198). Valparaíso: Ediciones Universitarias de Valparaíso.

Piqué-Noguera, C. (2012). Writing business research article abstracts: a genre approach. Ibérica. Journal of the European Association of Languages for Specific Purposes, 24, 211-232.

Samraj, B. (2002). Introductions in research articles: variation across disciplines. English for Specific Purposes, 21, 1-7. doi:10.1016/S0889-4906(00)00023-5

Samraj, B. (2005). An exploration of a genre set: Research article abstracts and introductions in two disciplines. English for Specific Purposes, 24, 141-156. doi: 10.1016/j.esp.2002.10.001

Sánchez, U. A. (2012). Análisis lingüístico de artículos de investigación en ciencias sociales y humanas. Lingüística y Literatura, 33(62), 105-121.

Santos, M. B. (1996). The textual organization of research paper abstracts in applied linguistics. Text, 16(4), 481-499. doi:10.1515/text.1.1996.16.4.481

Suntara, W., \& Usaha, S. (2013). Research article abstracts in two related disciplines: rhetorical variation between linguistics and applied linguistics. English Language Teaching, 6(2). doi:10.5539/elt.v6n2p84

Swales, J. (1981). Aspects of Article Introductions. Birmingham: University of Aston, Language Studies Unit.

Swales, J. M. (1990). Genre analysis: English in academic and research setting. Cambridge: Cambridge University Press.

Swales, J. (2004). Research genres: explorations and applications. Cambridge: Cambridge University Press.

Swales, J. M., \& Feak, C. B. (2009). Abstracts and the writing of abstracts. Ann Arbor MI.: The University of Michigan Press.

Weissberg, R., \& Buker, S. (1990). Writing up research. Experimental research report writing for students of English. Englewood Cliffs, NJ.: Prentice Hall Regents. 